\title{
COINFECCIÓN POR Mycobacterium abscessus Y Mycobacterium tuberculosis EN UN PACIENTE CON SÍNDROME DE CUSHING EXÓGENO Y OTRAS COMORBILIDADES
}

\author{
Gino Patrón-Ordóñez@1,2,a , Félix Llanos-Tejada (1),2,b, Dayanne Benites-Gamboa®1,c, \\ César Espinoza-Chiong ${ }^{1,0}$ \\ 1 Facultad de Medicina Humana, Universidad Ricardo Palma, Lima, Perú. \\ 2 Hospital Nacional Dos de Mayo, Lima, Perú. \\ a Médico internista; ${ }^{\mathrm{b}}$ médico neumólogo; ${ }^{\mathrm{c}}$ médica/o cirujana/o
}

\section{RESUMEN}

Mycobacterium abscessus es una micobacteria no tuberculosa de crecimiento rápido, que produce infección pulmonar, cutánea, diseminada y otras, sobre todo en pacientes con comorbilidades. El diagnóstico se basa en la identificación de la micobacteria por medios de cultivo o por pruebas moleculares. El tratamiento con macrólidos y amikacina continúa siendo el ideal, aunque depende de la localización y de la gravedad de la infección, sin embargo, se han identificado genes de resistencia en algunas subespecies que limitan la eficacia antibiótica. Presentamos el caso de un paciente con coinfección cutánea por Mycobacterium abscessus y Mycobacterium tuberculosis, quien presenta un síndrome de Cushing exógeno, factor predisponente para estas infecciones. Además, se identificaron hidatidosis y aspergilosis pulmonares. El tratamiento antituberculoso del paciente se ajustó para manejar ambas micobacterias, y su evolución fue favorable.

Palabras clave: Micobacterias no tuberculosas; Mycobacterium abscessus; Mycobacterium tuberculosis; tuberculosis pulmonar; síndrome de Cushing (Fuente: DeCS BIREME).

\section{Mycobacterium abscessus AND Mycobacterium tuberculosis COINFECTION IN A PATIENT WITH EXOGENOUS CUSHING SYNDROME AND OTHER COMORBILITIES}

\begin{abstract}
Mycobacterium abscessus is a fast-growing non-tuberculous mycobacterium that causes lung, skin, disseminated and other infections, mainly in patients with comorbidities. The diagnosis is based on the identification of the mycobacterium by culture media or molecular tests. Treatment with macrolides and amikacin remains the optimal choice, although it depends on the location and severity of the infection; however, resistance genes have been identified in some subspecies that limit antibiotic efficacy. We present the case of a patient with cutaneous coinfection by Mycobacterium abscessus and Mycobacterium tuberculosis, who presented exogenous Cushing syndrome, a predisposing factor for these infections. In addition, hydatidosis and pulmonary aspergillosis were identified. The patient's anti-tuberculosis treatment was adjusted to manage both mycobacteria, resulting in a favorable evolution.
\end{abstract}

Keywords: Nontuberculous mycobacteria; Mycobacterium abscessus; Mycobacterium tuberculosis; pulmonary tuberculosis; Cushing syndrome (Source: MeSH NLM).

\section{INTRODUCCIÓN}

Correspondencia: Gino Patrón Ordóñez; Facultad de Medicina Humana, Universidad Ricardo Palma, Av. Alfredo Benavides 5440, Santiago de Surco, Lima, Perú; g.patron@urp.edu.pe

El género Mycobacterium se caracteriza por estar conformado de bacilos inmóviles y dependientes de oxígeno. Se clasifica según sus características en el complejo Mycobacterium tuberculosis, Mycobacterium leprae y en micobacterias no tuberculosas o atípicas. Estas bacterias producen cuadros clínicos muy variados, como compromiso pulmonar, linfático, sistémico y cutáneo, y dentro de este último el Mycobacterium abscessus produce lesiones como pápulas, pústulas, abscesos y nódulos cutáneos ${ }^{(1,2)}$. 
El síndrome de Cushing o hipercortisolismo crónico produce alteraciones en el sistema inmune, que frecuentemente acarrea complicaciones clínicas graves como sepsis e infecciones oportunistas; asimismo, la administración de dosis elevadas de glucocorticoides durante dos a cuatro semanas es un factor de riesgo para presentar otras formas de tuberculosis ${ }^{(3,4)}$.

Se presenta el caso de un paciente con síndrome de Cushing exógeno y tuberculosis pulmonar, hospitalizado por síntomas respiratorios y lesiones cutáneas nodulares de aspecto esporotricoide, cuyo diagnóstico etiológico fue Mycobacterium abscessus. Durante su evolución, se añadieron los diagnósticos de hidatidosis y aspergilosis pulmonar, patologías no reportadas como coinfecciones en pacientes con micobacteriosis atípica. El presente reporte sigue las recomendaciones de la guía CARE (Case Report) ${ }^{(5)}$

\section{REPORTE DE CASO}

Varón de 59 años, procedente de Junín (Perú); en los últimos diez años trabajó en la minería, agricultura y en el comercio de frutas; presentó episodios recurrentes de hemoptisis leve a moderada, la más intensa fue hace dos años. Acudió al hospital regional, donde luego de varios estudios y procedimientos se le diagnostica hidatidosis pulmonar. Adicionalmente se le realiza un aspirado bronquial por broncofibroscopia, cuyo resultado del cultivo se desconoce, ya que el paciente no continuó con sus controles.

Permaneció durante dos años con episodios recurrentes de hemoptisis y se automedicó con prednisona $20 \mathrm{mg}$ diarios. Refirió mejoría hasta que presentó un cuadro de hemoptisis moderada, motivo por el cual acudió al Servicio de Emergencia del Hospital Nacional Dos de Mayo.

Al examen clínico, se encontraba hemodinámicamente estable y con rostro pletórico. Se observó un aumento de volumen en la zona cervical posterior, en forma de "giba de búfalo», con tejido celular subcutáneo aumentado a predominio centrípeto y edemas moderados en los cuatro miembros. Estos hallazgos clínicos son compatibles con el síndrome de Cushing exógeno debido a la automedicación con corticoides. Además, tenía varias lesiones nodulares flogóticas en el antebrazo y en el dorso de mano derecha, algunas de consistencia blanda y otras gomosas con pequeñas úlceras centrales que drenaban secreción seropurulenta. Dichas lesiones tenían un patrón esporotricoide con cierto trayecto lineal, como una linfangitis, que le causaban leve dolor (Figura 1). El paciente refirió que las lesiones habían aparecido hacía algunos meses, sin precisar fecha ni haber tenido un traumatismo previo.

En la auscultación pulmonar, se percibió roncus y un murmullo vesicular disminuido en hemitórax derecho. En la tomografía torácica se observó una imagen quística de contenido hidroaéreo e irregular en el lóbulo inferior derecho, compatible con un quiste hidatídico complicado y aspergiloma, además se hallaron bronquiectasias, tractos fibróticos, calcificaciones

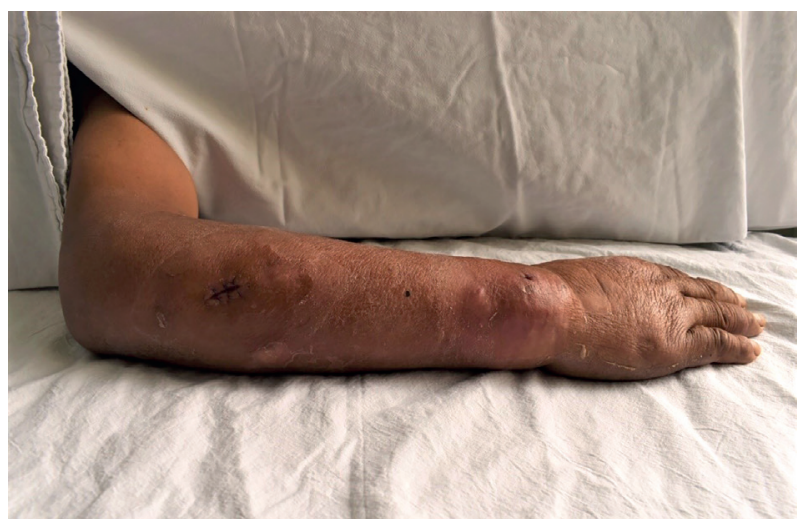

Figura 1. Miembro superior derecho con lesiones nodulares flogóticas que siguen un trayecto lineal y que tienden a formar fístulas.

parenquimales, ganglios mediastinales calcificados, engrosamiento pleural derecho y múltiples nódulos de aspecto de vidrio esmerilado (Figura 2). Estos hallazgos son indicativos de secuelas y actividad de tuberculosis pulmonar.

En las pruebas de laboratorio se encontró un valor de hemoglobina de $12,7 \mathrm{~g} / \mathrm{dL}$, leucocitos y plaquetas normales, que se mantuvieron sin mayor variación durante toda hospitalización. Asimismo, los valores de glucosa, urea, creatinina y el perfil de coagulación siempre se mantuvieron normales.

Con todos los hallazgos se propusieron los diagnósticos diferenciales de tuberculosis cutánea, infección por micobacterias atípicas y esporotricosis. Se realizó el examen directo, el cultivo de la secreción de la herida y la biopsia de la piel; el resultado fue positivo a la tinción de Ziehl-Neelsen y la biopsia que se le practicó mostró una dermatitis crónica abscedada con presencia de micobacterias (Figura 3). Se diagnosticó tuberculosis cutánea y se inició terapia antibiótica con isoniacida, rifampicina, pirazinamida y etambutol, y quedó pendiente el cultivo y la determinación de la sensibilidad.

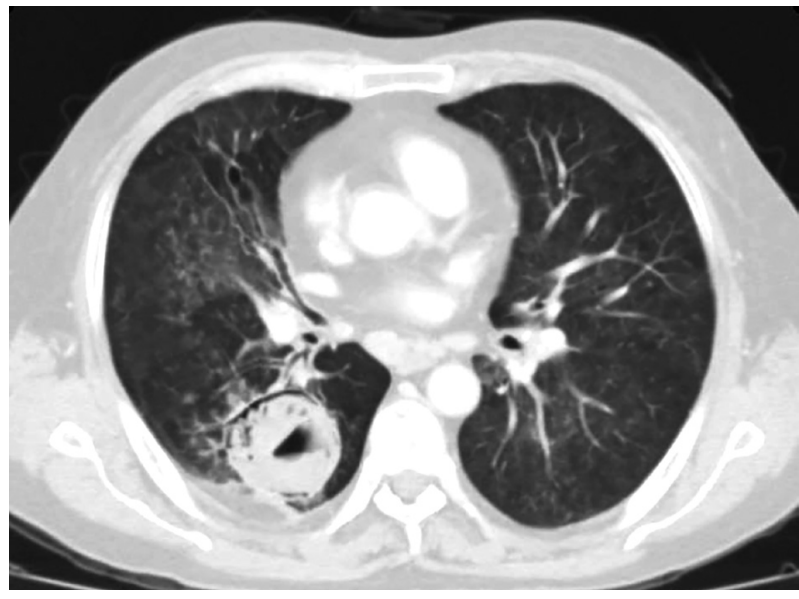

Figura 2. Tomografía torácica. Se observa una imagen cavitaria de contenido irregular, sugerente de quiste hidatídico complicado que contiene aspergiloma. 


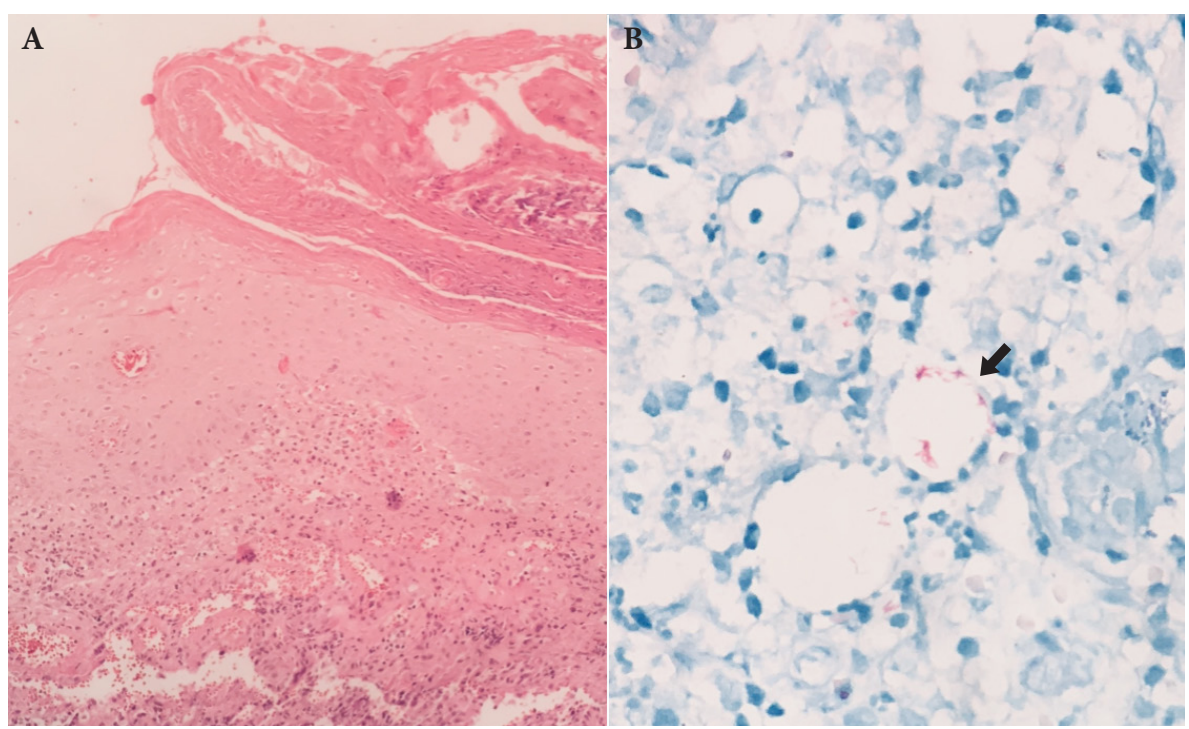

Figura 3. Biopsia de piel. A) Aumento 10×. Coloración hematoxilina-eosina. Dermatitis crónica con infiltrado mononuclear y células multinucleadas en la dermis, no se observa necrosis caseosa ni formación de granulomas. B) Aumento 100×. Coloración de Ziehl-Neelsen. Grupos de bacilos ácido-alcohol resistentes en la dermis (flecha).

Se accedió al portal NetLab (https://www.netlab.ins.gob. pe) del Instituto Nacional de Salud para buscar el resultado del cultivo obtenido por broncofibroscopía de hace dos años. Este resultado fue positivo en medio BACTEC MIGT para Mycobacterium tuberculosis pansensible, lo que concuerda con los hallazgos tomográficos descritos, y confirma el diagnóstico de tuberculosis pulmonar.

Adicionalmente, se realizó la prueba de Western-Blot para hidatidosis, la cual resultó positiva, confirmándose así el diagnóstico. También se le indica una broncofibroscopía, cuyo cultivo del aspirado bronquial dio positivo para Aspergillus fumigatus, lo que confirmó la sospecha de aspergiloma. Se indicó cirugía de tórax para realizar una lobectomía.

Durante el tratamiento, la evolución de las lesiones cutáneas no fue favorable, existía la posibilidad de una tuberculosis drogorresistente. Sin embargo, la prueba Genotype ${ }^{\circledR}$ MTBDRplus realizada en la secreción de la herida identificó al Mycobacterium abscessus. Con estos resultados y debido a que el paciente presentó hipertransaminasemia y un cuadro de náuseas y vómitos, concordante con una reacción adversa a fármacos antituberculosos, se replanteó el esquema de tratamiento de la siguiente manera: claritromicina, amikacina, etambutol, cicloserina y ciprofloxacino. Posteriormente se agregaron isoniacida y rifampicina. Con este esquema, las lesiones del paciente mejoraron de manera progresiva.

El paciente se sometió a una lobectomía, los hallazgos operatorios fueron una masa de $10 \mathrm{~cm} \times 15 \mathrm{~cm}$ en el segmento seis de pulmón derecho, con membranas hidatídicas y un aspergiloma, lo cual se corroboró en el estudio anatomopatológico.

El paciente evolucionó de forma satisfactoria y fue dado de alta hospitalaria con diagnósticos de infección cutánea por Mycobacterium abscessus, tuberculosis pulmonar, hidatidosis pulmonar, aspergiloma y síndrome de Cushing exógeno, con un tratamiento ambulatorio con isoniacida, rifampicina, etambutol y claritromicina. Continuó con sus controles y completó el tratamiento, sin evidencia de reactivación de la enfermedad.

\section{DISCUSIÓN}

Las infecciones por micobacterias causadas por especies distintas al complejo Mycobacterium tuberculosis y Mycobacterium leprae son conocidas como infecciones por micobacterias atípicas o no tuberculosas. Se clasifican en cuatro grupos según su crecimiento, lento (menos de 7 días) o rápido (más de 7 días), y según la capacidad de pigmentación de sus colonias (Tabla 1),

Tabla 1. Micobacterias no tuberculosas de interés dermatológico

\begin{tabular}{ll}
\hline Grupos & Especies más frecuentes \\
\hline I & \\
Fotocromógenas & Mycobacterium kansasii \\
Pigmentadas solo a la luz & Mycobacterium marinum \\
De crecimiento lento & \\
II & Mycobacterium scrofulaceum \\
Escotocromógenas & Mycobacterium szulgai \\
Pigmentadas con o sin luz & Mycobacterium gordonae \\
De crecimiento lento & \\
III & Mycobacterium avium-intracellulare \\
No cromógenas & Mycobacterium haemophilum \\
No pigmentadas & Mycobacterium ulcerans \\
De crecimiento lento & \\
IV & Mycobacterium fortuitum \\
De crecimiento rápido & Mycobacterium chelonae \\
\hline
\end{tabular}


produciendo cuadros clínicos en su mayoría de compromiso pulmonar, ganglionar, cutáneo y también diseminado, sobre todo en pacientes con comorbilidades ${ }^{(1,2,6)}$.

De esa clasificación, las más asociadas a infecciones cutáneas son las micobacterias de crecimiento rápido del grupo IV, donde se encuentran Mycobacterium fortuitum, Mycobacterium chelonae y Mycobacterium abscessus, además de las micobacterias de otros grupos como Mycobacterium marinum y Mycobacterium ulcerans ${ }^{(6-8)}$.

La taxonomía actual refiere que el complejo Mycobacterium abscessus tiene tres subespecies: Mycobacterium abscessus, Mycobacterium massiliense y Mycobacterium bolletii, que es el menos frecuente; la diferenciación de estas subespecies es difícil aun con pruebas genotípicas ${ }^{(1,9)}$. Estas micobacterias producen infecciones pulmonares, cutáneas, oculares, diseminadas, del sistema nervioso y de catéteres, en pacientes con distintas comorbilidades, incluido el síndrome de Cushing; pero no se han reportado coinfecciones con tuberculosis pulmonar, aspergilosis o hidatidosis ${ }^{(1,10,11)}$, estos dos últimos no se describen como factores predisponentes para infecciones por micobacterias. En el paciente, las coinfecciones estarían favorecidas por las secuelas pulmonares de tuberculosis que predisponen al aspergiloma y por la procedencia de un área endémica de hidatidosis.

La infección cutánea por Mycobacterium abscessus produce pápulas, pústulas, abscesos o nódulos cutáneos de patrón esporotricoide, localizados principalmente en los miembros inferiores y es frecuente en sujetos que reciben corticoterapia, como en el presente caso. Los mecanismos patogénicos son la inoculación y la diseminación de la enfermedad ${ }^{(2,6,10)}$. Existen reportes de asociación con tratamientos cosméticos, acupuntura y cirugías que podrían favorecer la entrada de la micobacteria ${ }^{(12-14)}$. Se debe considerar que el paciente ha desempeñado ocupaciones que podrían relacionarse con una posible inoculación.

Los pacientes con síndrome de Cushing sufren alteraciones en el sistema inmune durante la fase activa y de remisión. En la fase activa el aumento de la exposición a glucocorticoides causa directamente la supresión en el sistema inmunitario, alterando todas las vías de la respuesta inmune y expone a los pacientes a un mayor riesgo de diseminación sistémica y a infecciones adquiridas u oportunistas. Los patógenos responsables de las infecciones oportunistas más frecuentemente asociados al síndrome de Cushing son Pneumocystis, Cryptococcus, Pseudomonas aeruginosa, Nocardia y Klebsiella pneumoniae. Sin embargo, también se han reportado como agentes oportunistas a las micobacterias y se reconoce que la administración de $15 \mathrm{mg} /$ día o más de prednisona durante dos a cuatro semanas es un factor de riesgo para presentar tuberculosis ${ }^{(3,4,15)}$, tal como presumiblemente sucedió en este caso.

El tratamiento recomendado para infecciones por Mycobacterium abscessus se basa en series de casos, donde se reporta alta resistencia a la mayoría de los antibióticos, siendo los macrólidos (claritromicina o azitromicina) y los agentes parenterales (amikacina, cefoxitina, imipenem) el tratamiento de elección, ambos se administran juntos, por al menos dos semanas, y luego solo macrólidos ${ }^{(1,9,10,16-19)}$, tal como se procedió en este caso.

Ante un paciente con lesiones gomosas cutáneas se debe incluir como diagnóstico diferencial la infección por micobacterias, sin embargo, el tratamiento no debe basarse solo en los resultados de la tinción de Ziehl-Neelsen positiva, ya que esta prueba no distingue entre micobacterias tuberculosas y no tuberculosas. Estas micobacterias se deben identificar con cultivos o pruebas moleculares, aun con un diagnóstico confirmado de tuberculosis pulmonar, ya que puede existir coinfección, sobre todo en un contexto de inmunosupresión.

Criterios éticos: Los autores declaran que el paciente brindó su consentimiento para el reporte del caso, además se respetó su privacidad y se obtuvieron los permisos correspondientes de la institución para la publicación del reporte.

Contribuciones de los autores: GPO concibió el artículo. GPO, DBG y CEC recolectaron datos y redactaron el artículo. GPO y FLT se encargaron de la revisión crítica del manuscrito y aprobaron su versión final.

Conflictos de interés: Los autores declaran no tener filiación con ninguna organización, industria o entidad que tenga algún interés financiero o no financiero con el tema o materiales mencionados en este manuscrito.

Financiamiento: El presente trabajo fue autofinanciado.

\section{REFERENCIAS BIBLIOGRÁFICAS}

1. Lee MR, Sheng WH, Hung CC, Yu CJ, Lee LN, Hsueh PR. Mycobacterium abscessus Complex Infections in Humans. Emerg Infect Dis. 2015; 21(9):1638-46. doi: 10.3201/2109.141634.

2. Alcaide F, Esteban J. Cutaneous and soft skin infections due to non-tuberculous mycobacteria. Enferm Infecc Microbiol Clin. 2010; 28 Suppl 1:46-50. doi: 10.1016/S0213-005X(10)70008-2.

3. Hasenmajer V, Sbardella E, Sciarra F, Minnetti M, Isidori AM, Venneri MA. The Immune System in Cushing's Syndrome. Trends Endocrinol Metab. 2020;S1043-2760(20)30081-3. doi: 10.1016/j.tem.2020.04.004.

4. Long W, Cai F, Wang X, Zheng N, Wu R. High risk of activation of latent tuberculosis infection in rheumatic disease patients. Infect Dis (Lond). 2020;52(2):80-86. doi: 10.1080/23744235.2019.1682187.
5. Rison RA, Kidd MR, Koch CA. The CARE (CAse REport) guidelines and the standardization of case reports. J Med Case Rep. 2013;7:261. doi: 10.1186/1752-1947-7-261.

6. Benwill JL, Wallace RJ. Mycobacterium abscessus: Challenges in diagnosis and treatment. Curr Opin Infect Dis. 2014; 27(6):506-10. doi: 10.1097/ QCO.0000000000000104.

7. Bhambri S, Bhambri A, Del Rosso JQ. Atypical mycobacterial cutaneous infections. Dermatol Clin. 2009; 27(1):63-73. doi: 10.1016/j.det.2008.07.009.

8. Griffith DE, Aksamit T, Brown-Elliott BA, Catanzaro A, Daley C, Gordin $\mathrm{F}$, et al. An official ATS/IDSA statement: diagnosis, treatment, and prevention of nontuberculous mycobacterial diseases. Am J Respir Crit Care Med. 2007; 175(4):367-416. doi: 10.1164/rccm.200604-571ST. 
9. Tung-Chen Y, Carballo-Cardona C. Infección por micobacterias no tuberculosas tras cirugía estética. Med Clínica. 2017; 149(5):227-8. doi: 10.1016/j.medcli.2017.03.017.

10. Nessar R, Cambau E, Reyrat JM, Murray A, Gicquel B. Mycobacterium abscessus: a new antibiotic nightmare. J Antimicrob Chemother. 2012; 67(4):810-8. doi: 10.1093/jac/dkr578.

11. Novosad SA, Beekmann SE, Polgreen PM, Mackey K, Winthrop KL. Treatment of Mycobacterium abscessus infection. Emerging infectious diseases. 2016; 22(3):511. doi: 10.3201/eid2203.150828.

12. Lamb RC, Dawn G. Cutaneous non-tuberculous mycobacterial infections. Int J Dermatol. 2014; 53(10):1197-204. doi: 10.1111/ijd.12528.

13. Bello L, Vázquez P, Rodríguez I, Pedreira JD. Infección diseminada por mycobacterium abscessus en paciente infectado por el VIH. Enferm Infecc Microbiol Clinica. 2012:47-8. doi: 10.1016/j.eimc.2011.07.012.

14. Lee WJ, Kang SM, Sung H, Won CH, Chang SE, Lee MW, et al. Non-tuberculous mycobacterial infections of the skin: a retrospective study of 29 cases. J Dermatol. 2010; 37(11):965-72. doi: 10.1111/j.13468138.2010.00960.x.
15. Haas SR, Hodge MB, Duncan RA. Cushing's syndrome presenting as disseminated cutaneous Mycobacterium chelonae infection. Clin Infect Dis. 2001;33(6):e51-e53. doi:10.1086/322629.

16. Wongkitisophon P, Rattanakaemakorn P, Tanrattanakorn S, Vachiramon V. Cutaneous Mycobacterium abscessus Infection Associated with Mesotherapy Injection. Case Rep Dermatol. 2011;3 (1):37-41. doi: $10.1159 / 000324766$

17. Koh WJ, Jeon K, Lee NY, Kim BJ, Kook YH, Lee SH, et al. Clinical significance of differentiation of Mycobacterium massiliense from Mycobacterium abscessus. Am J Respir Crit Care Med. 2011; 183(3):405-10. doi: 10.1164/rccm.201003-0395OC.

18. Huang YC, Liu MF, Shen GH, Lin CF, Kao CC, Liu PY, et al. Clinical outcome of Mycobacterium abscessus infection and antimicrobial susceptibility testing. J Microbiol Immunol Infect. 2010; 43(5):401-6. doi: 10.1016/S1684-1182(10)60063-1.

19. Choi WS, Kim MJ, Park DW, Son SW, Yoon YK, Song T, et al. Clarithromycin and amikacin vs. clarithromycin and moxifloxacin for the treatment of post-acupuncture cutaneous infections due to Mycobacterium abscessus: a prospective observational study. Clin Microbiol Infect. 2011;17(7):1084-90. doi: 10.1111/j.1469-0691.2010.03395.x. 